Article

\title{
This Might Hurt a Little: Finding Common Ground in Vaccine Hesitancy
}

\author{
February 17, 2021
}

Lessons from past failure

A common adage among people who study the concept of vaccine hesitancy is that vaccines are "a victim of their own success." In other words, vaccines have been so successful at eliminating infectious diseases that people's ability to assess the risk associated with these diseases has been muted. Lacking any access to a first-hand understanding of the devastation of diseases like measles and polio, people have effectively ceased to view them as a risk and in many cases have begun to view the vaccines as at best unnecessary and at worst too risky to accept.

Or so the argument goes. In the midst of the Covid-19 outbreak, as it became clearer and clearer-in part because of our failure to change the course of the disease by consistently adopting social distancing and other measures-that the success of the US response to the pandemic depended heavily on the robust uptake of a vaccine, there was suddenly a lot of speculation from researchers and journalists about how people traditionally labeled as "antivaxxers" would react to a novel vaccine for a virus that was very much alive and well. Unlike vaccines for diseases like diphtheria and tetanus, with which most people do not tend to come into too much contact, the threat of Covid-19 was, and is, very real. Would so-called anti-vaxxers change their tune and accept the vaccine for an illness that was much more salient? Of course, if we accept the notion that vaccine hesitancy is largely caused by a kind of collective amnesia about the dangers of largely extinct diseases, it would seem that a vaccine for an illness that is currently ravaging the population would be more acceptable.

This is, however, not quite what has happened. Skepticism of Covid-19 vaccines has been vibrant. In a December study, about 40 percent of Americans said they would not get a vaccine due to a variety of concerns about safety and efficacy. The fact that there are actually multiple vaccines complicates communications about their safety and efficacy even further. At points, opposition to vaccines has become mired in politics, such as when those advocating "anti-vaccine" views started showing up at anti-lockdown protests and demanding their freedom be restored. Conspiracy theories about microchips being put into the vaccine by Bill Gates to track the population have also been advanced, although these 
fringe ideas are less commonly cited as a primary barrier to vaccination.

So what went wrong with the many predictions that the Covid-19 vaccine would be more acceptable to people than vaccines against diseases that have faded into the background? Before answering this question, it's worth looking at a historical example in which our predictions about acceptance of a new vaccine were wrong. In 2006, Gardasil, a vaccine that prevents human papilloma virus (HPV) infection, was approved for medical use in the US. In the years following, more and more medical entities recommended universal vaccination for both boys and girls, not least because the vaccine prevents several types of cancer. Given the cancer risk of HPV infection and the good efficacy and safety profile of the vaccine, no one expected any pushback, despite the fact that we were dealing with a sexually transmitted illness in the teenage population and that anti-vaccine sentiment was on the rise. Merck, the manufacturer of Gardasil, aggressively marketed the vaccine with no qualms about general distrust of big pharma in the population.

What followed was a major public health failure. Uptake of Gardasil is still abysmally low in the US despite repeated efforts on the part of public health agencies, physician groups, and others to convince people to get the vaccine. In 2016, Merck even came out with new ads featuring child actors asking their parents if they knew about the Gardasil vaccine with voiceovers revealing that these children later developed cancer due to HPV. The campaign met with anger from some viewers.

So what went wrong with Gardasil? The story is very similar to what is going wrong with our predictions about the population's acceptance of the coronavirus vaccine. Public health officials and policymakers are assuming a monolithic, homogeneous population and a single category of anti-vaccine attitudes rather than the complex patchwork that actually makes up a variety of reasons why people become hesitant to vaccinate themselves or their children. In this essay, I will discuss what happens when we oversimplify the world of vaccine hesitancy and how to better understand this patchwork and speak to the variegated audiences of people who fall into vaccine-hesitant beliefs. There are obvious implications here for how we message around the new Covid-19 vaccines, which could make a significant difference in the proportion of the population that agrees to get the shot. Equally important is to consider the question: If we stop viewing everyone with some level of vaccine hesitancy as a virulent anti-vaxxer, might we start to see more common ground?

\section{What's in a name?}

First, a note on language. In 2015, Jenny McCarthy, a celebrity and one of the foremost advocates of the debunked notion that vaccines cause autism, insisted in a PBS Frontline interview that people who hold this belief should not be called "anti-vaccine" but rather 
"pro-safe-vaccine-schedule." While this name might not actually be the best representation of McCarthy and her followers' views on vaccines, she raises an important point about framing. How we label people, especially people with whom we may disagree, is extremely important, not only to how they respond to us but also to how we go about approaching them in the first place. If we are "pro-vaccine" and we view everyone who doubts or questions vaccines as "anti-vaccine," then our stance is immediately combative. Yet in many cases, this may not be an accurate reflection of the situation, and there may indeed be far more common ground than we think.

In a similar vein, in December 2019, a vaccine-skeptical group called "Crazymothers" released a statement requesting that the media retire the use of the term "anti-vaxx" and replace it with the term "vaccine risk aware." In their statement on Twitter, they claimed that the term "anti-vaxx" is "derogatory, inflammatory, and marginalizes both women and their experiences." The problem with the proposed name "vaccine risk aware" is that it suggests both that vaccines are particularly risky, which they tend not to be, and that others, including those in the medical establishment, are by contrast somehow "unaware" of any risks associated with vaccines, which is also misleading and could cause diminished trust in health-care workers in the context of making vaccination decisions. Nonetheless, we once again see that names make a difference.

Many years of excessive focus on a group of people called "anti-vaxxers" has also led to a lack of focus on people who are mildly hesitant, are on the fence, or even accept vaccines now but may change their minds later if the circumstances around a particular vaccine cause a heightened perception of risk (as is the case with the Covid-19 vaccines-we will come back to this later). As a result, we spend much of our energy studying and trying to convince a very small number of people who are adamant that vaccines are dangerous and not nearly enough time understanding the underlying conditions that cause hesitancy to arise and what tools can be used to reinforce the safety and efficacy of vaccines even among people who currently do not view them as particularly risky. We in turn spend even less time addressing legitimate issues of trust in the health-care system, including in communities of color, in which distrust is even more understandable given ongoing patterns of systemic inequities and mistreatment of these populations in the health-care system.

\section{Is there an anti-vaccine "movement"?}

One reason it may be difficult to figure out how to refer to people with high levels of skepticism about vaccines is a lack of clarity surrounding whether anti-vaccine and vaccinehesitant sentiments constitute a "movement." It is extremely common to see the phrases "anti-vaccine movement" and "pro-vaccine movement" in media coverage, including in headlines, as in "How pro-vaccine parents can help stop the rise of the anti-vaxxer 
Despite the second headline's insistence that the so-called movement is growing during the pandemic, it would seem that Covid-19 has actually called into question any sense of an organized, monolithic group of people driving anti-vaccine sentiment and vaccine hesitancy. For one thing, the "anti-vaxxers" are not behaving the way we thought they would. Instead of being humbled by the obvious need for a vaccine to control a raging epidemic, antivaccine sentiments have flourished in this environment. Among the American public more generally, people were much more skeptical of a potential Covid-19 vaccine than anticipated. The thought went that everyone would be so desperate for this crisis to end that they would rush to get vaccinated, but polls asking people about their plans once a vaccine became available did not bear this out. In every instance, researchers were very surprised to find that large quantities of people are either not planning to get the vaccine or want to significantly delay getting it.

The reality is that vaccine hesitancy is not that uncommon and encompasses a wide spectrum of views, from total acceptance to total rejection. Framing "anti-vaxxers" as a movement can actually help the cause of the most extreme vaccine skeptics by making them seem like a unified group. People feel comfortable when they join groups-it is a very socially desirable activity and helps make difficult decisions, like whether or not to get a brand-new vaccine whose long-term consequences have not been assessed, easier. Without meaning to, when we repeatedly refer to the "anti-vaxx movement," we may be pushing people toward more extreme views rather than helping communities address the specific and varied concerns they might have. We are robbing ourselves of an opportunity to better understand a rich spectrum of views and instead focusing on a vocal fringe that is not going to engage with us much anyway.

\section{Who are the "anti-vaxxers"?}

If a monolithic view of the "anti-vaccine movement" is misguided, then who are the socalled anti-vaxxers? Who is most vulnerable to becoming "vaccine hesitant," and how do we understand the different categories of people within this designation?

While the "categories" of people who tend toward hesitancy are by no means absolute, it is somewhat helpful to think of the different types of objections to vaccines if we want to be effective at targeting and correcting these views. While people who claim that vaccines are part of a vast government conspiracy may be the most visible and vocal, vaccine hesitancy consists of a wide array of concerns and barriers, many of which are actually quite mundane. This is important because if we always focus on the flashy phenomena such as conspiracy theories and direct all of our messages there, we will miss entire populations of 
people who may have more common, understandable concerns-concerns that we may be able to address with respectful and constructive dialogue. That's why a careful assessment of different kinds of vaccine-hesitant sentiments is so important. And it's also another reason why conceptualizing a monolithic "anti-vaccine movement" is so counterproductive.

A recent study suggested four different "types" of so-called anti-vaxxers, which the authors called the four Cs: complacency, convenience, lack of confidence, and utility calculation. Complacency refers to a sense that the diseases against which vaccines protect are not really a threat; convenience refers to the kinds of structural barriers, such as time and cost, that might keep people from prioritizing and following through with a vaccine; lack of confidence refers to what we most commonly think of when we hear the term "antivaxxer" - that is, a sense that the safety of the vaccine is in question; and utility calculation refers to the notion that people may not bother getting vaccinated because they think they are protected by others in the population who are immune. Other studies have suggested similar formulations of hesitancies.

\section{Is coronavirus vaccine hesitancy different?}

While it is too soon to fully understand how hesitancy around Covid-19 vaccines will play out, there is still some basis to speculate on how it might compare to the kind of vaccine hesitancy we have been seeing over the past few decades. On the one hand, there are a lot of similarities between Covid-19 vaccine hesitancy and hesitancy surrounding other vaccines. We often hear a lot of the same arguments meant to discredit the need to get a vaccine, including the supposed superiority of natural immunity over vaccine-acquired immunity, the idea that the pharmaceutical industry is pushing something unnecessary and potentially unsafe for its own benefit, and the argument, which comes up a lot around the seasonal flu vaccine, that the disease is not that bad.

On the other hand, hesitancy around Covid-19 vaccines is actually quite different from hesitancy around established vaccines. In some ways, the argument is less polarized, as some trepidation around a new vaccine is quite logical. There are understandable concerns, although they are probably ultimately unfounded, about the speed with which Covid-19 vaccines have been developed. It is not completely unreasonable for people to be nervous about a new vaccine when we have no longer-term information on how it might affect people. Against a backdrop of decreasing trust in government authorities, which is also understandable given many of the actions of the current outgoing administration, hesitancy around a new vaccine developed with hefty support from the government is not exactly surprising or even wholly unwarranted.

How hesitancy around Covid-19 vaccines will ultimately play out is going to function as an 
interesting natural experiment on how vaccine hesitancy develops and flourishes. In this case, researchers and policymakers actually have the opportunity to watch hesitancy unfold as the vaccines are developed and distributed in real time. Hopefully, if we pay enough attention, we will learn something about which efforts and messages work and don't work to convince people that the vaccines are safe and effective and to reach reasonable levels of population immunity.

\section{Where do we go from here?}

Vaccine hesitancy is certainly not a one-size-fits-all phenomenon, and if anything it has only become more complex with the new threat of Covid-19 and accompanying vaccines. As we struggle to keep hesitancy at bay in the coming months and years, it's essential to continue to search for common ground. One of the best ways to find it is to fully embrace the fact that vaccine hesitancy is a heterogeneous phenomenon. This understanding will undoubtedly have implications for how we actually address vaccine hesitancy and look to persuade people to accept vaccines for Covid-19 and other diseases. But no matter what method of persuasion and behavior change we choose, it is fair to say that the way we conceptualize vaccine hesitancy has a significant impact on how we respond to it. If we are going to be successful at vaccinating the public against this novel threat, we need to reframe both our language and our concept of the so-called "anti-vaxxer." 